\title{
STUDY OF DIFFRACTIVE BREMSSTRAHLUNG AT 13 TeV LHC*
}

\author{
SABina CZEKIERdA \\ on behalf of the Cracow AFP Group \\ The Henryk Niewodniczański Institute of Nuclear Physics \\ Polish Academy of Sciences \\ Radzikowskiego 152, 31-342 Kraków, Poland
}

(Received April 7, 2016)

\begin{abstract}
Feasibility studies of the diffractive bremsstrahlung measurement at the LHC at $\sqrt{s}=13 \mathrm{TeV}$ are presented. The method considered for this measurement uses the ATLAS detector and, in particular, the Zero Degree Calorimeter and the ATLAS Forward Proton detectors. The signal and background processes were generated with GenEx and PYTHIA 8.2 generators, respectively. The obtained fiducial cross sections are $1.2 \mu \mathrm{b}$ for the signal and $6 \mu \mathrm{b}$ for the background. Further reduction of the background is possible by the optimisation of event selection cuts.
\end{abstract}

DOI:10.5506/APhysPolB.47.1639

\section{Introduction}

Bremmstrahlung is an electromagnetic radiation produced by a moving charged particle as the result of its deflection from the original trajectory. It can be emitted during the proton-proton elastic scattering in the reaction

$$
p+p \rightarrow p+p+\gamma
$$

Based on the type of a particle exchanged between the interacting protons, two possibilities can be distinguished. The first one is the electromagnetic bremsstrahlung where a photon is the exchanged particle (Fig. 1(a)). The second one is the diffractive bremsstrahlung, where a Pomeron mediates the interaction (Fig. 1(b)).

* Presented at the Cracow Epiphany Conference on the Physics in LHC Run 2, Kraków, Poland, January 7-9, 2016. 

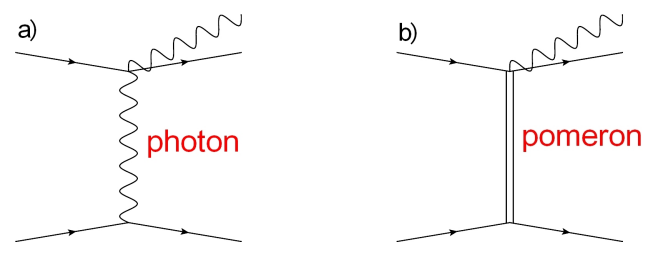

Fig. 1. Feynman diagrams of the (a) electromagnetic, (b) diffractive bremsstrahlung.

At the centre-of-mass energy $\sqrt{s}=13 \mathrm{TeV}$, in the range of radiated photon energy between $100 \mathrm{GeV}$ and $1500 \mathrm{GeV}$, the integrated cross section is about $50 \mathrm{nb}$ for the electromagnetic bremsstrahlung and about $2.8 \mu \mathrm{b}$ for the diffractive one, so the Pomeron exchange is the prevailing mechanism.

The angular distribution of radiated photons is driven by the Lorentz factor of emitting particle [1], here a proton, $\gamma_{p}=E_{p} / m_{p}$. The average photon emission angle is $\left\langle\theta_{p}\right\rangle \approx 1 / \gamma_{p}$ and at the $\sqrt{s}=13 \mathrm{TeV}$, it is about $10^{-4}$ rad or equivalently the pseudorapidity ${ }^{1}|\eta| \approx 14$, so the bremsstrahlung radiation is collimated in the forward directions. Owing to the properties of the exchange, in both discussed cases, the sum of the photon and the scattered proton energies is approximately equal to the radiating particle energy and, at the collider, it is $E_{\text {beam }} \approx E_{\gamma}+E_{p}$.

\section{Method of measurement}

The method of the measurement presented below uses the ATLAS experiment apparatus [2], but it could be also applied to CMS and TOTEM experiments.

Bremsstrahlung process in $p p$ collisions is characterized by a very simple final state - two protons and a photon. This is shown in a simplified way in Fig. 2 together with the experimental set-up sketch.

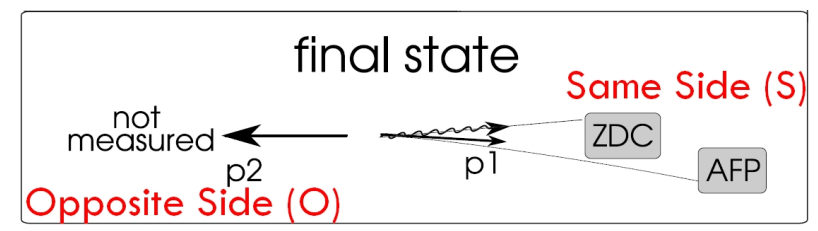

Fig. 2. Experimental set-up to register bremsstrahlung.

\footnotetext{
${ }^{1}$ The pseudorapidity is defined as $\eta=-\ln \tan \Theta / 2$, where $\Theta$ is the particle polar angle.
} 
The reaction can be divided into hemispheres. The one containing a proton and a photon is the signal hemisphere, denoted with " $\mathrm{S}$ ". The other one is denoted with "O". A proton and a photon belonging to the signal hemisphere can be registered by the AFP [3] and the ZDC [4], respectively.

The AFP detector will measure the forward scattered protons and is dedicated to the diffractive physics studies. It uses the Roman Pot stations situated $210 \mathrm{~m}$ from the interaction point. It allows the detection of protons with the relative energy loss, $\xi=\left(E_{\text {beam }}-E\right) / E_{\text {beam }}$, ranging from 0.02 to 0.12 , so the registration of a proton belonging to the "O" hemisphere is excluded. The detector delivers the scattered proton trajectory position which can be used to reconstruct the proton energy with $10 \mathrm{GeV}$ resolution [5].

The photon energy can be measured by the electromagnetic module of the Zero Degree Calorimeter dedicated to the detection of neutral particles, and which is situated 140 meters from the interaction point. The ZDC face is rectangular, with the dimensions of $91.4 \mathrm{~mm}$ in horizontal and $180 \mathrm{~mm}$ in vertical direction, respectively. It covers the range of $|\eta|>8.3$, therefore, the vast majority of the bremsstrahlung photons can be detected by the ZDC. For a single photon, the ZDC spatial resolution is about $0.5 \mathrm{~mm}$, while the energy resolution was parametrised according to [4].

\section{Analysis}

Diffractive bremsstrahlung events were generated using the GenEx generator [6]. The signal signature is the electromagnetic energy deposit in the ZDC accompanied by a scattered proton registered in the AFP. Owing to the fact that the exchanged particle is soft, the sum of the reconstructed photon and proton energies, $E_{\mathrm{ZDC}, \mathrm{EM}_{\mathrm{S}}}+E_{\mathrm{AFP}_{\mathrm{S}}}$, should be close to the incident proton energy. Moreover, only one particle should be reconstructed in the electromagnetic part of the ZDC.

The distribution of the sum of the reconstructed photon and proton energies belonging to the "S" hemisphere, $E_{\mathrm{ZDC}, \mathrm{EM}_{\mathrm{S}}}+E_{\mathrm{AFP}_{\mathrm{S}}}$, is illustrated in Fig. 3 (left) for events containing a photon with the energy between $100 \mathrm{GeV}$ and $1500 \mathrm{GeV}$ and a proton within the ZDC acceptance. The maximum of this distribution corresponds to the beam energy and the RMS is $20.3 \mathrm{GeV}$. The distribution of the photon position reconstructed from the ZDC measurement is shown in Fig. 3 (right). There is a clear peak in the central region and beyond it, the distribution steeply falls with increasing distance from the calorimeter centre.

The requirement $\left|E_{\text {beam }}-E_{\mathrm{ZDC}, \mathrm{EM}_{\mathrm{S}}}-E_{\mathrm{AFP}_{\mathrm{S}}}\right|<60.9 \mathrm{GeV}$ is fulfilled by about $43 \%$ of events, which corresponds to the signal fiducial cross section of about $1.2 \mu \mathrm{b}$. 

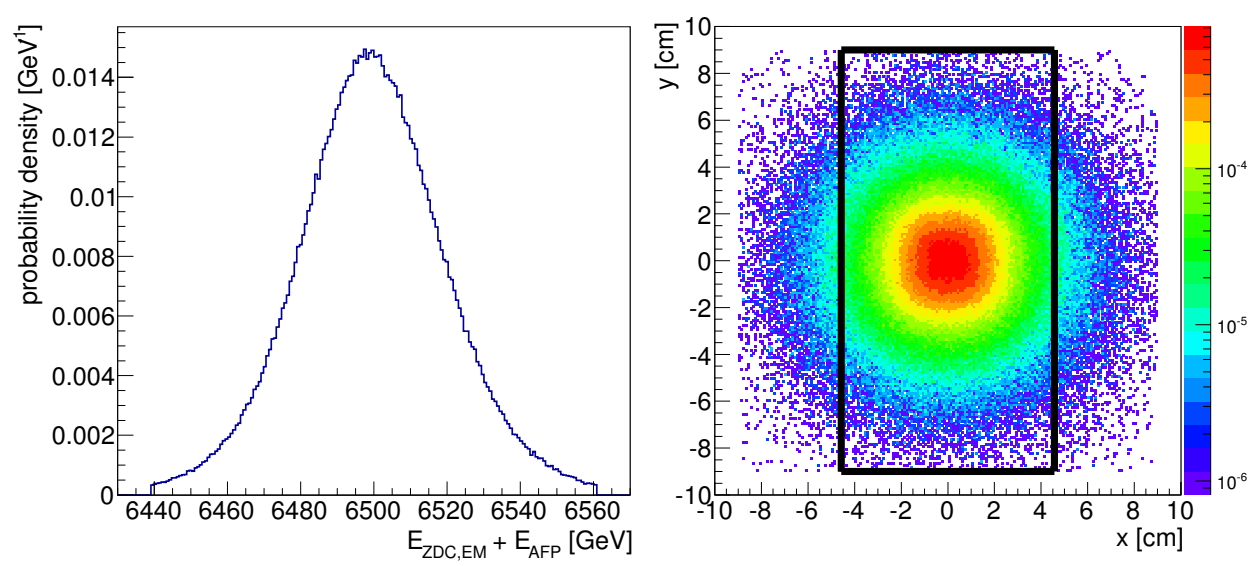

Fig. 3. Signal studies. Left: $E_{\mathrm{ZDC}_{\mathrm{E}} \mathrm{EM}_{\mathrm{S}}}+E_{\mathrm{AFP}_{\mathrm{S}}}$ probability distribution. Right: photon position probability distribution at the ZDC (the black rectangle shows the ZDC face contour).

\section{Background}

To study the feasibility of the measurement, the background processes have to be considered. In the present analysis, the background study was carried out using PYTHIA 8.2 [7]. Single, double and central diffractive processes were generated. The PYTHIA predicted cross section for these processes is $\sim 22 \mathrm{mb}$ at $\sqrt{s}=13 \mathrm{TeV}$.

A background event has to imitate the signal signature in the detector acceptance - a single photon in the ZDC and a proton in the AFP. Therefore, an event was rejected from the analysis if it contained a charged particle within the Inner Detector acceptance $(|\eta|<2.5$ and the transverse momentum $p_{\mathrm{t}}>0.5 \mathrm{GeV}$ ) or a particle with the energy $E>1 \mathrm{GeV}$ and $|\eta|<4.8$ (the calorimeter acceptance).

For the remaining events, the ZDC response was calculated. The multiphoton events were rejected if the maximum distance between the reconstructed positions of the photons was larger than $6 \mathrm{~mm}$. Otherwise, the sum of the reconstructed energies was treated as resulting from a single photon impact. The next requirement was that the events passing the above selection contain a single, forward produced proton with the energy within the AFP acceptance.

Summarising, the accepted background events were characterised by the following properties:

- there was a single proton emitted into the "S" hemisphere with the energy within the AFP acceptance, 
- there was only "one" photon reconstructed in the ZDC with the energy between $100 \mathrm{GeV}$ and $1500 \mathrm{GeV}$,

- the sum of the proton and photon reconstructed energies fulfilled the approximated energy conservation

$$
\left|E_{\mathrm{AFP}_{\mathrm{S}}}+E_{\mathrm{ZDC}, \mathrm{EM}_{\mathrm{S}}}-E_{\text {beam }}\right|<60.9 \mathrm{GeV},
$$

- the electromagnetic energy reconstructed in the ZDC in the "O" hemisphere $E_{\mathrm{ZDC}, \mathrm{EM}_{\mathrm{O}}}<10 \mathrm{GeV}$,

- the energies reconstructed in the hadronic parts of the ZDC had to be below $50 \mathrm{GeV}$ in order to suppress the background processes with a forward neutron.

The main background source that satisfies the above requirements is the reaction

$$
p+p \rightarrow p+p+\pi^{0}
$$

in which one photon from the $\pi^{0}$ decay is within the ZDC acceptance.

In Fig. 4 (left), the sum of the photon and proton energies, $E_{\mathrm{ZDC}, \mathrm{EM}_{\mathrm{S}}}+$ $E_{\mathrm{AFP}_{\mathrm{S}}}$ for background events, is shown. This distribution has a completely different shape from that observed for the signal (Fig. 3 (left)). There is no maximum at the beam energy. Instead, the distribution is shifted towards the lower values, which demonstrates that a part of the total energy is carried by particles escaping the detection. Figure 4 (right) presents the distribution of the photon position at the ZDC face for the background events. In
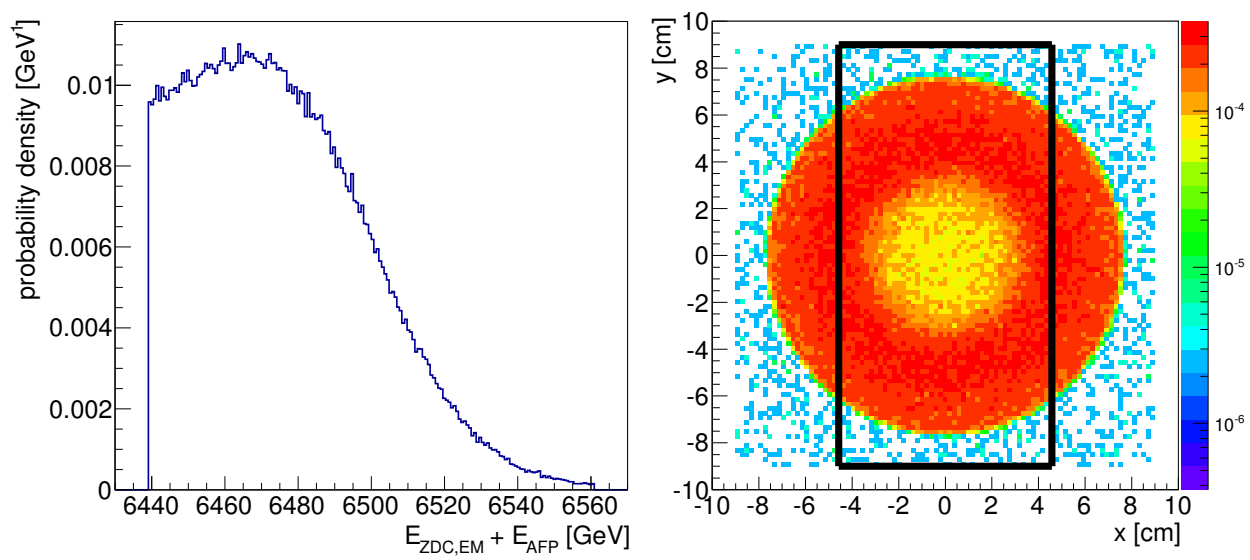

Fig. 4. Background studies. Left: $E_{\mathrm{ZDC}, \mathrm{EM}_{\mathrm{S}}}+E_{\mathrm{AFP}_{\mathrm{S}}}$ probability distribution. Right: photon position probability distribution at the ZDC (the black rectangle shows the ZDC face contour). 
contrast to the distribution for the signal, there is no peak at the central part and the distribution for the background reaches the maximum and is nearly flat for $3 \mathrm{~cm} \leq \sqrt{x^{2}+y^{2}} \leq 7 \mathrm{~cm}$.

About $27 \%$ of the background events pass the selection criteria hence, the fiducial cross section of the background processes is about $6 \mu \mathrm{b}$.

\section{Conclusions and outlook}

Two types of bremsstrahlung processes were discussed. In high-energy $p p$ collisions, the prevailing mechanism is the exchange of a Pomeron. At the centre-of-mass energy of $13 \mathrm{TeV}$, the diffractive bremsstrahlung cross section is of the order of $2.8 \mu \mathrm{b}$, while the electromagnetic one - about $50 \mathrm{nb}$ if the radiated photon energy is between $100 \mathrm{GeV}$ and $1500 \mathrm{GeV}$. It was postulated to register the diffractive bremsstrahlung events using the ATLAS ZDC and AFP detectors. The estimated fiducial cross section is about $1.2 \mu \mathrm{b}$.

The background processes were also discussed and it was found that the main source is the $p p \rightarrow p p \pi^{0}$ process. The background fiducial cross section is about $6 \mu \mathrm{b}$. To perform the measurement, a further reduction of the background is required. This can be achieved by optimising the experimental veto on the background processes and by rejecting the background originating events according to the photon position reconstructed in the ZDC.

Additionally, the natural consequence of the described analysis will be an implementation of the proton transport, taking into account various possible optics of the LHC.

This work was supported in part by the Polish National Science Centre grant: UMO-2012/05/B/ST2/02480.

\section{REFERENCES}

[1] J.D. Jackson, Classical Electrodynamics, $3^{\text {rd }}$ ed., Wiley, New York 1999.

[2] ATLAS Collaboration, JINST 3, S08003 (2008).

[3] ATLAS Collaboration, Technical Design Report for the ATLAS Forward Proton Detector, CERN-LHCC-2015-009, ATLAS-TDR-024.

[4] ATLAS Collaboration, Letter of Intent, Zero Degree Calorimeters for ATLAS, CERN-LHCC-2007-01.

[5] R. Staszewski, J. Chwastowski, Nucl. Instrum. Methods Phys. Res. A 609, 136 (2009).

[6] R.A. Kycia et al., arXiv:1411.6035 [hep-ph].

[7] T. Sjöstrand et al., Comput. Phys. Commun. 191, 159 (2015). 